\title{
Étude des composantes du billan hydrique d'un sol par télédétection
}

\author{
Study of soil water balance components by teledetection
}

\author{
André Musy, Paul Meylan, Claude Morzier*
}

Ingénieurs EPF-L

\section{Introduction}

L'exploitation, la planification et la gestion de l'eau pour les besoins agricoles impliquent la connaissance du bilan hydrique du sol.

L'établissement de ce bilan fait appel soit à des anallyses rigoureuses, soit à des techniques empiriques. Cependant, le problème crucial de la représentativité d'une mesure localisée pour l'étude de l'ensemble d'une région n'est pas encore résolu. La variation spatiale des paramètres agro-météorologiques dépend en effet des relations et intéractions fort complexes existant entre le sol, l'atmosphère et la végétation. Seule la multiplication des mesures sur le terrain permet de mettre en évidence de telles variations, aux prix d'importantes difficultés économiques et d'exécution.

La télédétection peut apporter une solution à ce problème. En effet, la mesure de la radiance d'un sol et de sa couverture s'effectue, en télédétection, à partir d'une certaine altitude. Les appareils montés à bord d'avions ou de satellites peuvent capter et enregistrer dans un temps très court les radiations émises et/ou réfléchies par le sol sur l'ensemble d'un territoire.

Le problème de l'acquisition "spatiale" des données pouvant servir à l'analyse hydrologique d'une région est ainsi résolu. Il en soulève toutefois un second, et des plus importants ; celui de l'interprétation des données acquises par télédétection.

(*) Institut de Génie Rural, Ecole Polytechnique Fédérale de Lausanne (Suisse).

\section{Le bilan hydrique}

Il résulte du principe de la loi de la conservation de la matière appliquée à l'eau contenue dans le sol :

$$
P=R+I+E T \pm \Delta S
$$

avec

$P=$ précipitations

$R=$ ruissellement superficiel

$I \quad=$ infiltration

$E T=$ évapotranspiration

$\Delta S=$ variation des réserves en eau dans le sol

La quantité d'eau retenue par l'interception des végétaux est volontairement négligée dans cette équation. De plus, sur terrain plat, le terme de ruissellement est remplacé par celui de la rétention en surface de l'eau $(R T)$.

Détermination des composantes du bilan hydrique par télédétection

\section{Les précipitations}

L'analyse des données de télédétection ne permet pas encore d'estimer valablement la quantité d'eau qui va précipiter sur une région déterminée. L'estimation de ce paramètre continue donc d'être appréciée à l'aide de mesures conventionnelles. Remarquons que la précipitation est en général le paramètre le mieux connu dans une région. Sa détermination ne présente que peu de difficultés sur un territoire doté d'un réseau convenable d'observation. 


\section{L'évapotranspiration}

La transformation d'une certaine quantité d'eau en vapeur, soit par évaporation, soit encore par transpiration des végétaux est liée intimement au bilan radiatif. Aussi, plusieurs chercheurs ont-ils tenté d'exprimer l'évapotranspiration d'un bassin hydrologique en terme énergétique. Deux types de formules sont issus de cette analyse :

$$
E T R=R_{n}+R_{c}+R_{i}
$$

où

$E T R=$ évapotranspiration réelle

$R_{n} \quad$ = rayonnement net à la surface du sol

$R_{c} \quad=$ énergie convective (non radiative)

$R_{i} \quad=$ énergie emmagasinée par le sol, due à son inertie thermique

et :

$$
E T R=a+b R_{n}
$$

avec : $a$, et $b$ : coefficients d'ajustements fonction du type de couverture végétale, de la résistance stomatique et du micro-climat.

Ces deux expressions supposent que l'énergie de photosynthèse est négligeable.

La première tient compte de l'énergie de convection, que l'on ne sait pas mesurer à l'échelle d'un bassin, tandis que la seconde introduit des paramètres régionaux d'ajustement (qui en tiennent compte implicitement).

A l'échelle du bassin, et sous des conditions très restrictives d'homogénéité de couverture et d'uniformité de climat, certains auteurs égalent l'évapotranspiration réelle au rayonnement net :

$$
E T R=R_{n}
$$

Le rayonnement net d'une région peut être déterminé par télédétection. Le principe de cette mesure est décrit à la figure 1 .

- La radiance spectrale reçue par le radiomètre : (1) $R_{\lambda}^{r}$ est la somme de :

a) la radiance spectrale $R_{\lambda}^{g}$ de la cible au sol (2) atténuée par la couche atmosphérique (3) de coefficient de transmission $t_{3}(\lambda)$

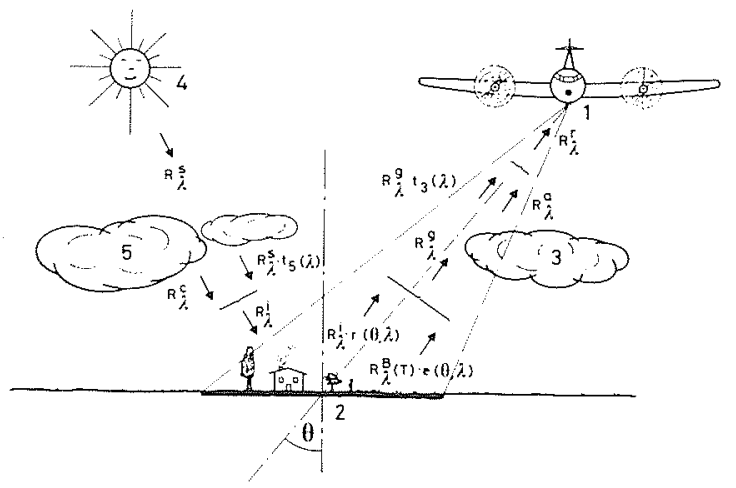

Figure 1. - Principe des mesures radiométriques b) la radiance spectrale $R_{\lambda}^{a}$ de l'atmosphère (3)

- La radiance spectrale $R_{\lambda}^{g}$ de la cible au sol (2), se compose de :

a) la radiance spectrale propre de la cible (émission thermique) $R_{\lambda}^{g} 1=R_{\lambda}^{B}(T) . e(\theta, \lambda)$

avec : $R_{\lambda}^{B}(T)$ radiance spectrale du corps noir à la température $T, e(\theta, \lambda)$ émissivité de la cible au sol

b) la radiance spectrale réfléchie par la cible en direction du capteur $R_{\lambda}^{g_{2}}=R_{\lambda}^{i} \cdot r(\theta, \lambda)$

avec : $R_{\lambda}^{i}$ radiance spectrale incidente sur la cible au sol, $r(\theta, \lambda)$ réflectivité de la cible au sol

- La radiance spectrale incidente sur la cible (2) est égale à :

a) la radiance spectrale solaire $R_{\lambda}^{s}$ (4) atténuée par l'atmosphère terrestre (5) de coefficient de transmission $t_{5}(\lambda)$

b) la radiance $R_{\lambda}^{c}$ de la voûte céleste et de l'atmosphère (5) due à la lumière solaire diffuse ou à l'émission thermique propre de l'atmosphère.

Dès lors, le rayonnement net d'une portion de sol se détermine par:

$$
R_{n}=R_{\lambda}^{i}-R_{\lambda}^{g}
$$

Les paramètres inconnus dans cette équation sont les coefficients de réflexion $r(\theta, \lambda)$ dans le domaine visible et d'émissivité $e(\theta, \lambda)$ dans celui de l'infrarouge. Ils sont toutefois mesurés implicitement par télédétection. Les autres facteurs intervenant dans le calcul du rayonnement net peuvent être calculés sous différentes conditions.

Ainsi, l'application d'une telle méthode permet d'estimer l'évapotranspiration réelle d'une région. Les travaux publiés à ce jour [6,7] montrent que cette technique, appliquée à de grandes surfaces, sous-estime en général l'ETR (de 30 à $50 \%$ ). Ce résultat n'est pas surprenant. Le terme convectif étant négligé dans le calcul de l'ETR, l'influence du vent n'est donc pas prise en considération.

\section{La variation de réserve en eau dans le sol}

Nous ne présentons ici que la variation correspondant à la zone non saturée du sol.

En télédétection, la détermination du taux d'humidité dans le sol n'est possible qu'à partir de mesures effectuées dans le domaine des hyperfréquences. En effet, les radiances observées dans les zones visible et infrarouge du spectre électromagnétique ne reflètent que des phénomènes de surface. Le pouvoir de pénétration du rayonnement dans ces 2 domaines est quasi nul (quelques microns pour l'infrarouge thermique !).

Les capteurs sensibles aux hyperfréquences mesurent, quant à eux, l'émission globale issue des premiers horizons du sol. (Epaisseur de l'ordre de grandeur de la longueur d'onde du rayonnement : $5-10 \mathrm{~cm}$ pour une fréquence de $10 \mathrm{GHz}, 20 \mathrm{~cm}$ environ pour une fréquence 
de $2 \mathrm{GHz}$ ). De plus, dans le domaine des ondes centimétriques, l'humidité du sol est le facteur primordial de la variation de l'émissivité.

L'Institut de Génie Rural de l'Ecole Polytechnique Fédérale de Lausanne et l'Institut de Physique Appliquée de l'Université de Berne ont entrepris en 1977 une campagne de mesure de l'humidité des sols à partir de données acquises dans le domaine des micro-ondes.

L'équipement radiométrique utilisé est constitué des appareils suivants :

- 4 radiomètres de fréquences différentes $(4.9,10.5$, $21,36 \mathrm{GHz}$ ).

- 1 thermomètre infrarouge $8-14 \mu$

- 2 caméras Hasselblad (émulsions couleur et fausse couleur).

L'ensemble de ces appareils, ainsi que leur électronique associée est localisé dans une caisse de mesure à température contrôlée, qui est soulevée à $15 \mathrm{~m}$ audessus du sol à l'aide d'une grue mobile.

Les mesures ont été effectuées sur terrain nu, afin de vérifier la possibilité de mesurer l'humidité du sol par télédétection, sans tenir compte de l'écran végétal.
Les conditions météorologiques ont été malheureusement peu favorables à une bonne dessication des sols. Ainsi le taux d'humidité pondérale en surface n'a varié que dans d'étroites limites durant les périodes de mesure : 30 à $45 \%$ à Vouvry, 10 à $23 \%$ à Changins, 50 à $65 \%$ et 36 à $44 \%$ à St-Aubin.

La figure 2 présente, à titre d'exemple, l'évolution dans le temps des paramètres principaux mesurés à Vouvry du 2.8 au 8.8.77.

Une première analyse sommaire des résultats fait apparaître une relation entre l'émissivité du sol, rapport entre la température de radiance à celle du sol, et l'humidité (cf. Figure 3). A Changins, pour une variation d'humidité de 8 à $25 \%$ et une fréquence de $4.9 \mathrm{GHz}$, la précision relative obtenue sur la détermination du taux d'humidité à partir d'une régression linéaire est en moyenne de $11 \%$ [10].

L'émission en micro-ondes peut être calculée à partir des profils d'humidité et de température du sol. En effet, à l'aide d'un modèle mathématique d'émission appliqué à un sol schématiquement divisé en différentes strates homogènes, il est possible de calculer la contribution radiative de chacune de ces strates. La

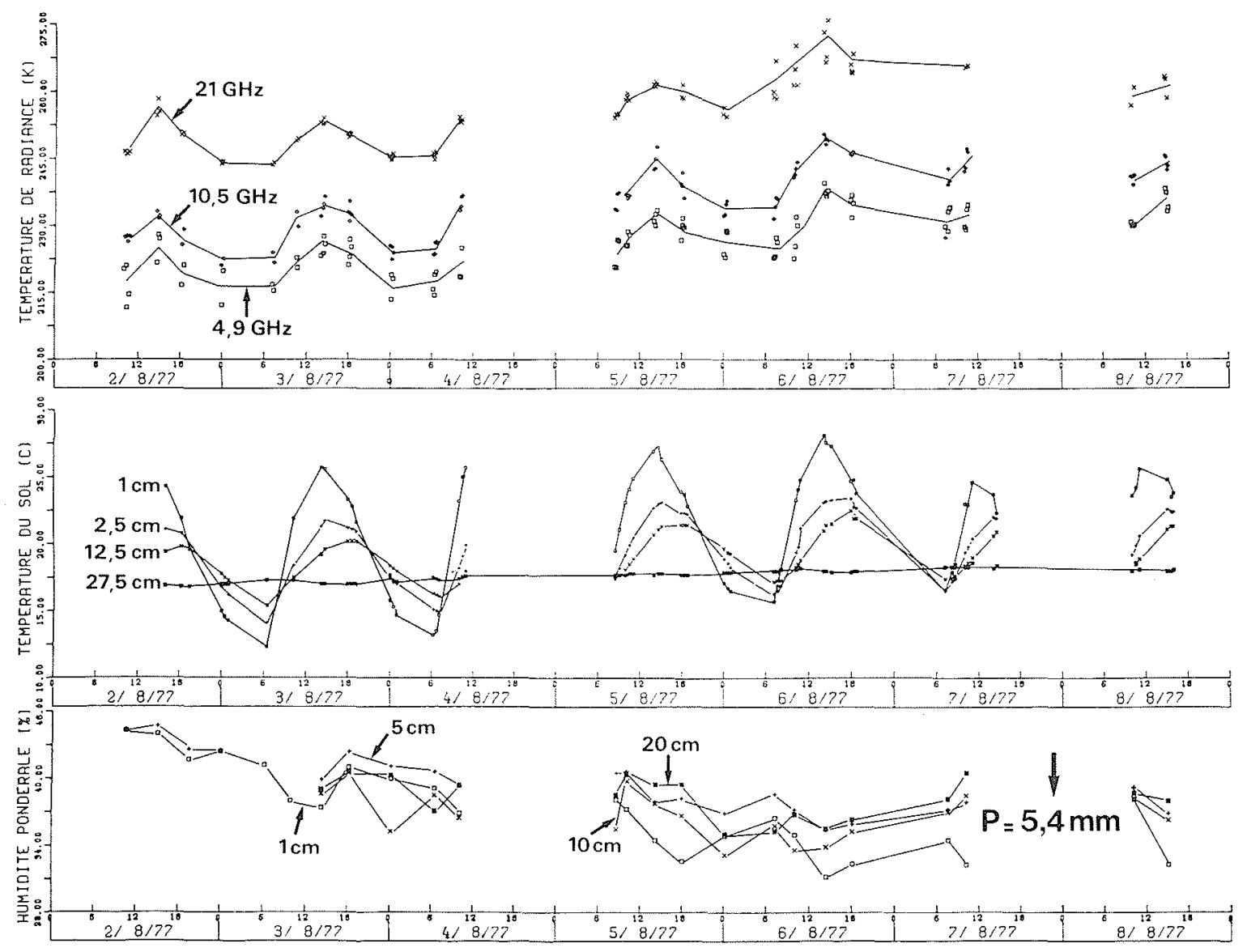

Figure 2 - Evolution des principaux éléments mesurés sur sol nu en fonction du temps. Vouvry 2.8.77/8.8.77 (limon, terrain hersé et roulé). 


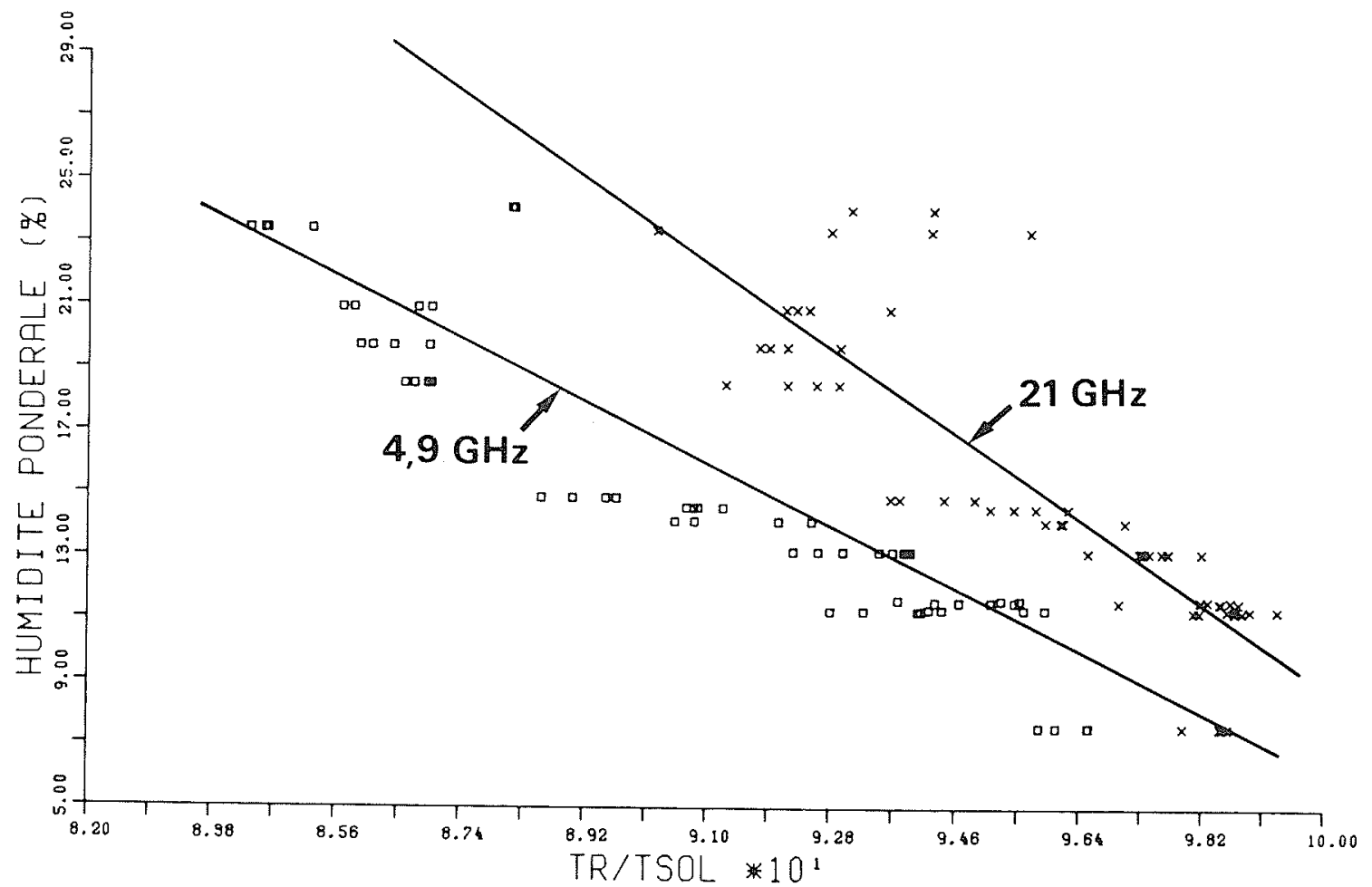

Figure 3 - Variation de l'humidité d'un sol en fonction de l'émissivité. Changins 22.8.77/2.9.77. (Limon argileux avec sable, traité par griffage).

radiance totale, mesurée par le capteur représente alors la somme de ces différentes contributions (cf. Fig. 4).

Nous avons tenté d'appliquer aux données de notre campagne de mesure le modèle d'émission proposé par Burke et Paris. Le calcul des températures de radiance conduit toujours à des valeurs plus faibles que celles mesurées. L'écart moyen entre ces températures de radiance est de l'ordre de grandeur de $50 \mathrm{~K}$. Cet écart s'explique de différentes manières, notamment :

- modèle de sol trop schématique, ne tenant pas compte de la rugosité,

- constantes diélectriques introduites mal adaptées aux différents sols

- influences atmosphériques négligées.

Ces différents facteurs peuvent être pris en compte en affinant ce type de modèle ou en appliquant un modèle d'émission plus souple.

Méthodologie générale pour l'étude spatiotemporelle des composantes du bilan hydrique d'un sol

\section{Les mesures conventionnelles}

L'étude du bilan hydrique d'une région implique la mise en cuvre de différentes mesures sur des parcelles témoins: les mesures agro-hydrologiques et météorologiques notamment. La fréquence de ces mesures varie beaucoup en fonction de l'accessibilité des zones test, le matériel et la main-d'œuvre disponibles.

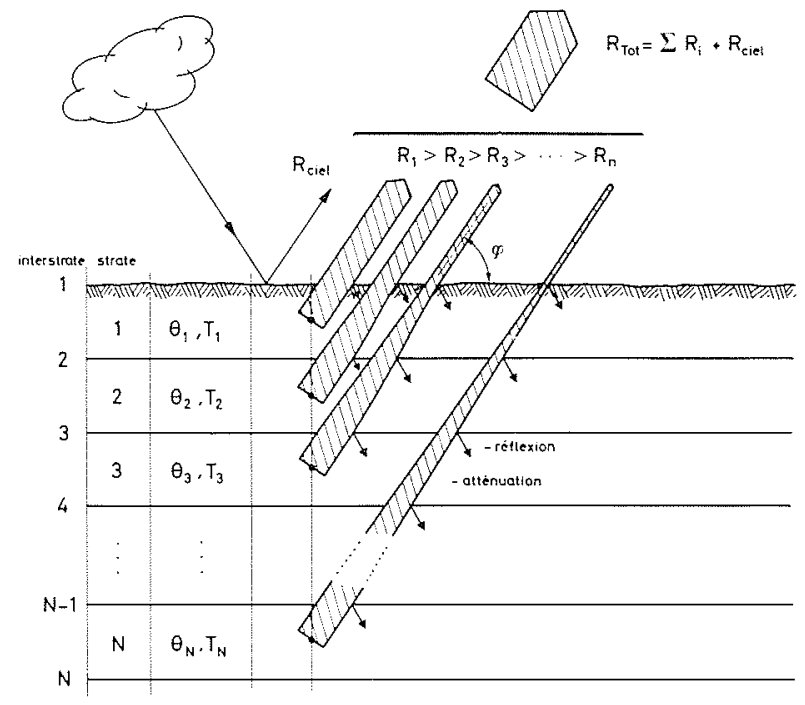

Figure 4 - Schématisation du modèle d'émission de Burke et Paris $[11]$.

Les données agro-pédologiques de base ne sont déterminées qu'une seule fois, en général. La météorologie, et plus spécialement certains de ses paramètres, sont connus journellement, voire même toutes les heures. Plus rares sont toutefois les mesures de la température et, surtout, de la teneur en eau d'un sol en différents points d'un territoire. 


\section{Les mesures de télédétection}

Par l'intermédiaire de systèmes montés à bord d'avion et de satellite, il sera possible d'estimer :

- le rayonnement net d'un sol (domaine visible + infrarouge)

- la température superficielle de ce sol (domaine IRT)

- la température de radiance du même sol (domaine micro-ondes).

La périodicité de ces mesures dépend actuellement essentiellement de la disponibilité d'un matériel très onéreux, ou de données peu facilement accessibles.

Cependant, et sur certaines régions, les mesures radiométriques sont en moyenne plus fréquentes que les données hydrodynamiques récoltées au sol. Aussi sont-elles utilisées pour l'ajustement de modèles de simulation ou pseudo-empirique qui tentent, entre 2 mesures in situ, de prévoir l'évolution temporelle de paramètres données $(\theta, T, E T)$.

\section{Evolution temporelle - méthodologie}

La technique d'analyse que l'Institut de Génie Rural se propose d'appliquer pour cette étude est présentée schématiquement à la figure 5 . Elle est basée sur les principes suivants :

- La mesure in situ est une mesure de référence. Elle sert au calage des modèles de simulation et au réajustement périodique des valeurs déterminées par d'autres moyens (simulation, télédétection). Sa fréquence est faible.

- Les modèles de simulation calculent l'évolution temporelle des données hydrodynamiques soit à partir de mesure in situ (si on en dispose) soit à partir de mesure de télédétection. Basés sur des données météorologiques journalières ou horaires, les calculs s'effectuent pour de courts intervalles de temps.

- Les mesures radiométriques acquises par télédétection servent à la correction des résultats obtenus par simulation. Les profils hydriques et thermiques sont ainsi réajustés après chaque passage d'avion ou de satellite sur la zone-témoin. La fréquence de ces vols est plus grande que celle des mesures in situ.

- Les résultats obtenus numériquement ou par télédétection sont confrontés périodiquement aux mesures au sol, ce qui permet de réajuster certains paramètres des modèles utilisés.

- L'ajustement du modèle de calcul ayant servi à la détermination de l'évapotranspiration par télédétection est effectué, compte tenu de l'état d'humidité du sol et des mesures météorologiques. Notons que les valeurs de l'ETR déterminées au sol à l'aide de formules classiques ne peuvent pas être prises comme références absolues!

- L'ajustement du profil hydrique à l'aide de données radiométriques micro-ondes (TR) est réalisé en utilisant un modèle d'émission (cf. Fig. 4), du type :

$$
T R=f(\theta, T, z)
$$

La détermination de $\theta$ en fonction de la profondeur $z$ est alors calculée, compte tenu de la mesure de $T R$ et du profil thermique corrigé $T(z)$, en résolvant implicitement l'équation (6).

Une telle méthode n'est pas immuable. Elle subira nécessairement certaines modifications, au fur et à mesure de sa mise en place, en fonction des expériences effectuées.

\section{Evolution spatiale}

Le modèle temporel décrit ci-dessus s'applique ponctuellement à de petites parcelles témoins situées à l'intérieur d'une région donnée. Il ne précise pas l'évolution spatiale des caractéristiques du sol sur l'ensemble d'un territoire. Celle-ci peut être toutefois obtenue de différentes manières:

- soit en interpolant ces caractéristiques à l'aide d'un algorithme connu

- soit en se basant sur les données spatiales recueillies par télédétection.

Cette dernière méthode semble être plus fiable que la première, car l'extrapolation des paramètres du sol est basée sur des mesures de radiance, élément de résolution par élément de résolution. Les relations établies pour chaque zone test entre les mesures radiométriques et les données au sol sont utilisées à cet effet.

\section{Conclusions}

La télédétection ne va remplacer ni les mesures in situ, ni les méthodes servant à la détermination des caractéristiques du sol. Son avantage réside principalement dans son caractère spatial et temporel. La télédétection offre en effet un intérêt scientifique voire même économique évident car, périodiquement, elle met à disposition de l'ingénieur, une série de mesures qui peut servir précisément à l'extrapolation de données et au contrôle systématique des caractéristiques du sol. Elle implique, en revanche, une méthodologie que certains trouvent trop compliquée en regard du bénéfice que l'on peut en tirer. N'oublions pas, toutefois, que l'utilisation de la télédétection pour les besoins agricoles est très récente. Les méthodes d'analyse évoluent très rapidement, au fur et à mesure des perfectionnements techniques des appareils utilisés. Ce qui paraît compliqué aujourd'hui le sera peut-être beaucoup moins demain.

De toute évidence, par télédétection on ne pourra pas dans un proche avenir, mesurer valablement le taux d'humidité dans un sol à partir d'une certaine profondeur $(>30-50 \mathrm{~cm})$. Il est également illusoire de penser que des mesures in situ pourront être effectuées systématiquement sur l'ensemble d'un territoire d'une certaine importance. Dès lors, l'utilisation d'un système hybride -mesure in situ - simulation - télédétection - s'avère indispensable pour une meilleure connaissance hydrologique de toute une région. L'utilisation rationnelle de l'eau pour les besoins agricoles est à ce prix. 


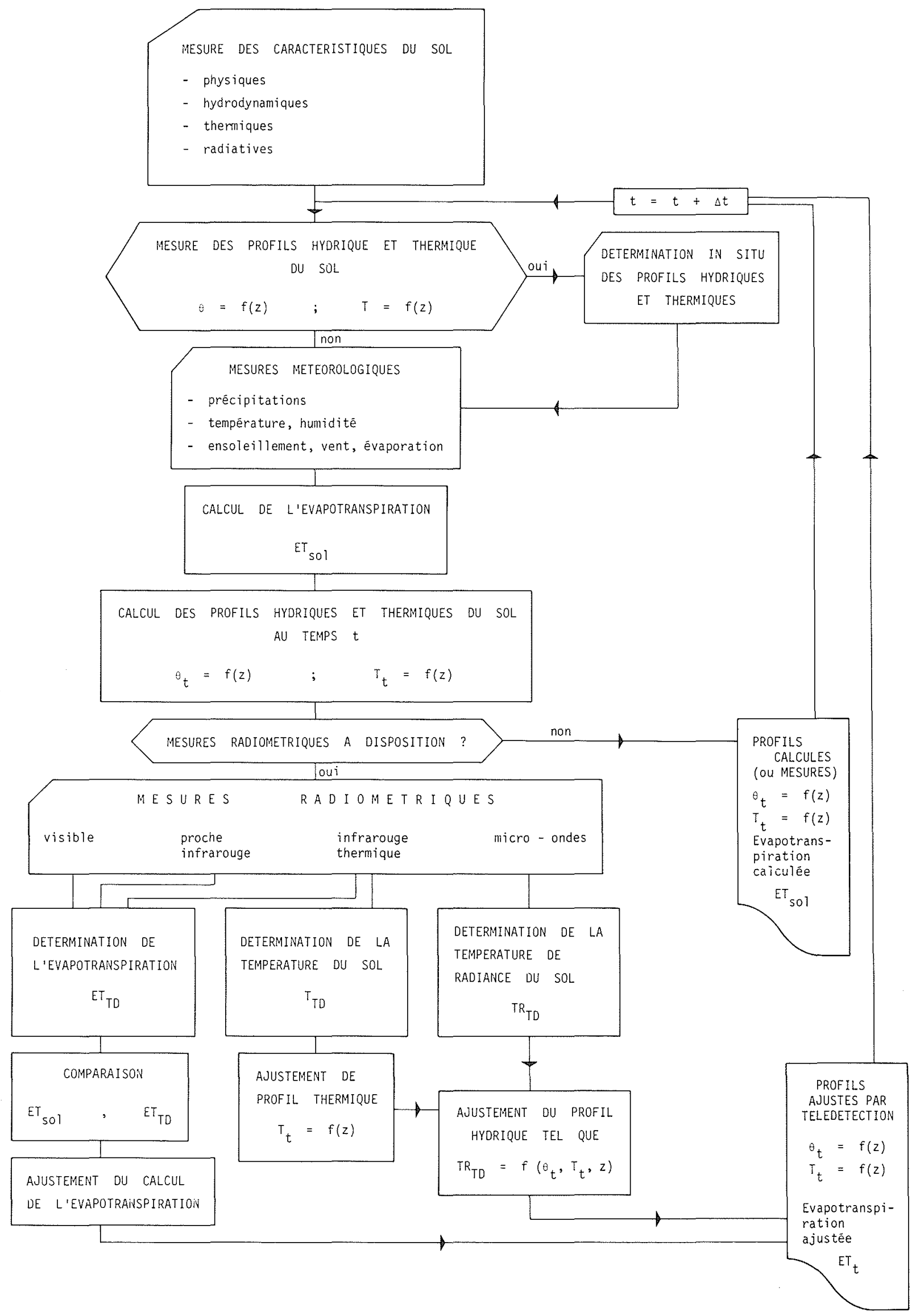

Figure 5 - Détermination par télédétection de l'évolution temporelle des composantes du bilan hydrique d'un sol. Schéma de principe. 


\section{Remerciements}

Cette étude n'aurait pas pu être entreprise sans : - le concours financier du Fonds National Suisse de la Recherche Scientifique

- le matériel et l'aide technique de l'Institut de Physique Appliquée de l'Université de Berne, notamment la collaboration du Professeur Schanda et de ses assistants, MM. Wyssen et Hofer

- les terrains d'essais que Ciba-Geigy et les Stations Fédérales de Recherches agronomiques mettent gratuitement à notre disposition.

Nous tenons à remercier chaleureusement ces différents organismes de leur aide précieuse.

\section{Références}

[1] MEYLAN P., MORZIER C. \& MUSY A. - Bases physiques de la télédétection - application à l'hydrodynamique des sols. I.G.R. No 132, Lausanne, Suisse, 1977.

[2] SCHANDA E. et al. - Remote Sensing for Environmental Sciences, Springer Verlag, 1976.
[3] REEVES R.G., ANSON A. \& LANDEN D. - Manual of Remote Sensing, American Society of Photogrammetry, 1975.

[4] BARRETT E.C. - Mapping rainfall from conventional data und weather satellite imagery across Algeria, Libya, Morocco and Tunisia, F.A.O. Rome, Jan. 1977.

[5] Measurement and Estimation of Evaporation and Evapotranspiration, Rapport O.M.M, No 201, 1966.

[6] HLAVEK R., OBERLIN G. et al. - Essai d'estimation de l'évapotranspiration réelle à l'échelle du bassin versant. Considérations théoriques et applications pratiques. CTGREF rapport No 1, avril 1974

[7] BOSSARD R., VUILLAUME Y. - Evaluation régionale de l'évapotranspiration et de l'humidité du sol - Possibilités d'emploi des radiomètres à infrarouge et à hyperfréquences du satellites NIMBUS 5, rapport BRGM, déc. 1975.

[8] BECKER F. - Infrared and microvaves techniques - Infrared thermography applications: Cours sur les Applications de la télédétection à l'agriculture et l'hydrologie, ISPRA, nov. 1977.

[9] MUSY A., MEYLAN P. \& MORZIER C. - Détermination de l'humidité du sol par télédétection. Colloque de l'Association Internationale de la science du sol, Rome, Italie, septembre 1977, I.G.R. No 137.

[10] MEYLAN P., MUSY A., MORZIER C., WYSSEN D. \& HOFER R. - Etude de la variation spatio-temporelle de l'humidité du sol par télédétection, Colloque OST, Toulouse, mars 1978.

[11] BURKE W.J. \& PARIS J.F. - A radiative transfer model for microwave emissions from bare agricultural soils, NASA TM X-58166/JSC-09836, 1976.

\section{Discussion}

Président : Professeur Dr. P. REGAMEY

Sur la proposition du Président, les mémoires successifs de MM. GIRARD et MUSY ont fait l'objet d'une discussion commune qu'il ouvre après avoir remercié les deux orateurs.

M. BECKER interroge d'abord M. MUSY sur la sensibilité de son modèle "humidité" aux erreurs commises sur la température de surface.

M. MUSY indique que si la corréiation entre les deux paramètres est relativement forte, le stade actuel de la mise au point des modèles ne lui permet pas de chiffrer avec précision leur sensibilité.

M. BECKER - Une erreur de $2 \%$ sur l'émissivité induit au moins un degré d'erreur sur la température.

Le président. - M. G IRARD a montré que la radiance n'était pas, ou était peu, influencée par les variations d'humidité lorsque le sol contient beaucoup de matière organique. Il devrait être possible de se dégager de ce "parasite" en faisant intervenir d'au tres canaux.

M. GIRARD - Pour s'affranchir de l'effet "matière organique", il faudra utiliser les réponses dans le "thermique" et dans les micro-ondes.

- La matière organique modifie la température des sols. Donc le "thermique" donnera des renseignements utiles.

- La matière organique intervient sur le modelé et la structure de la surface du sol. Les micro-ondes doivent aussi apporter des informations intéressantes.

Cependant la comparaison des informations obtenues dans les trois bandes de longueurs d'ondes : visible, thermique, microonde, risque d'être insuffisante. Il faut faire appel à la dynamique des phénomènes pour pouvoir différencier l'effet "matière organique" de l'effet "humidité" ; la matière organique donne des réponses constantes alors que l'humidité varie beaucoup plus. 


\section{Abstract \\ Study of soil water balance components by teledetection}

Various agrometeorological data are required for determining the water balance of soil in a given area, especially precipitation, evaporation and infiltration data. Teledetection can provide an answer for point measurement spatialization problems if the required parameters can be extracted from the teledetection data.

Soil water balance is given by formula (1).

Precipitation cannot yet be determined by teledetection.

Actual consumptive use can be found from the energy balance (formulae (2) and (3)) ; net radiation can be determined by teledetection (formula (5) and Fig. 1) ; the convective energy term $R_{c}$ has to be found by other methods.

Soil water storage variation can be determined by microwave teledetection, though only down to a limited depth (no deeper than $20-50 \mathrm{~cm}$ ).

French rural engineers of the "Institut de Génie Rural" have completed a programme of field measurements of soil moisture by hyperfrequency radiometry (e.g. Figs. 3 and 4).

The Burke and Paris radiative emission model has been tried and found to systematically give radiance temperatures below the measured values, probably owing to the use of dielectric constants determined in the laboratory for various typical soils and underestimation of reflected celestial radiance.

A hybrid model simulating the behaviour of the thermal and water profile of the ground is proposed (Fig. 5) as a means of "filling in the gaps" in teledetection and conventional measurement data. Its special features are as follows:

In-situ measurement data are used for reference purposes and to calibrate the model.

Profiles at time $t$ are calculated from weather data.

Occasional teledetection data are used for periodic correction of the calculated profiles.

This simulation is applied locally to a certain number of points in the considered area. The required parameters (e.g. soil moisture) are extended in space on the basis of the teledetection data, the relationship between radiometer measurements and soil data being established on the "simulation points". 